https://doi.org/10.29013/AJT-19-9.10-40-43

Timoshkin Andrey Ivanovich, candidate of physical and mathematical sciences, assistant professor National Metallurgical Academy of Ukraine Ukraine, Dnepr E-mail: timoshkin1964@gmail.com

\title{
TESTABLE LOGICAL CIRCUIT OF A BINARY NONRESTORING ARRAY DIVIDER
}

Abstract. In the information processing systems LSI with regular structure (adders, substractors, array multipliers, array dividers and so on) perform increasable part. A testable functional-logical circuit of a binary nonrestoring array divider has been elaborated. The circuit being represented possesses a fault detection test of the length of three and a small hardware complexity.

Keywords: logical circuits with regular structure, stuck-at fault, fault detection test.

It is easier to design testable digital integrated circuits with a regular structure, including adders, multipliers, substractors, dividers, and various memory circuits, than integrated circuits with an irregular structure. The article [1] presents a testable functional-logical circuit of a binary nonrestoring array divider with a fault detection test with a sequence length 4 or 5 for single stuck-at faults. However, its implementation requires a significant amount of hardware resources, which is a major flaw. This article proposes a testable functional-logical circuit of a binary nonrestoring array divider with a fault detection test with a sequence length 3 of the same fault class and with less hardware complexity.

Layers of 1-bit adders combined within each layer into parallel adder with sequential carry are the basis of this circuit. At the same time, the construction of a testable 1-bit adder circuit that underlies the multi-bit adder of each layer is based on the representation of the sum $S$ and carry $P$ functions of a full 1-bit adder as polynomials dual to the Zhegalkin polynomials [2, P. 24] (since the sum $S$ and carry $P$ functions are self-dual) and also on the fact that these polynomials for sum $S$ and carry $P$ functions of a 1-bit adder are simple:

$$
\begin{gathered}
P=(a \vee b) \odot(a \vee p) \odot(b \vee p), \\
S=\mathrm{a} \odot \mathrm{b} \odot \mathrm{p},
\end{gathered}
$$

where $\mathrm{a}$ and $\mathrm{b}$ are the values of the original 1-bit operands; $p$ and $P$ are the values of the input and output carry signals, respectively, $S$ is the value of the sum signal, $v$ is the symbol of the disjunction operation, $\odot$ is the symbol of the biconditional operation (equivalence).

The formula for the carry function P from (1) can easily be converted to convenient form: $P=(a \vee b) \odot[p \vee(a \odot b)]=\overline{(a \vee b)} \odot \overline{[p \vee(a} \odot \overline{b)}]$. (2)

Then the testable logical circuit of the 1-bit adder can be implemented in a logical basis consisting of NOR and EQUIVALENCE two-input gates. This diagram is shown in (Fig. 1). At the same time, a rather simple schematic diagram of EQUIVALENCE gate on MOS transistors, containing only 3 transistors [3, P. 196], is known. This scheme is shown in (Fig. 2). The fault detection test for the 1-bit adder circuits in the selected basis for all single stuck-at faults contains 3 vectors and is described in table shown in (Fig. 1).

The testable $n$-bit ( $\mathrm{n}$ is a positive integer) circuit of the binary adder of each layer of the nonrestoring array divider is composed of $\mathrm{n}$ testable circuits of a full 1-bit adder connected in a regular manner, i.e. by connecting the carry output of $i$-th circuit with the carry input of $(\mathrm{i}+1)$-th circuit, where $1 \leq i \leq n-1$. 
This circuit diagram is shown in (Fig. 3). A fault de- for all its single stuck-at faults is conducted by simply tection test for a testable circuit of n-bit binary adder iterating the test shown in (Fig. 1).

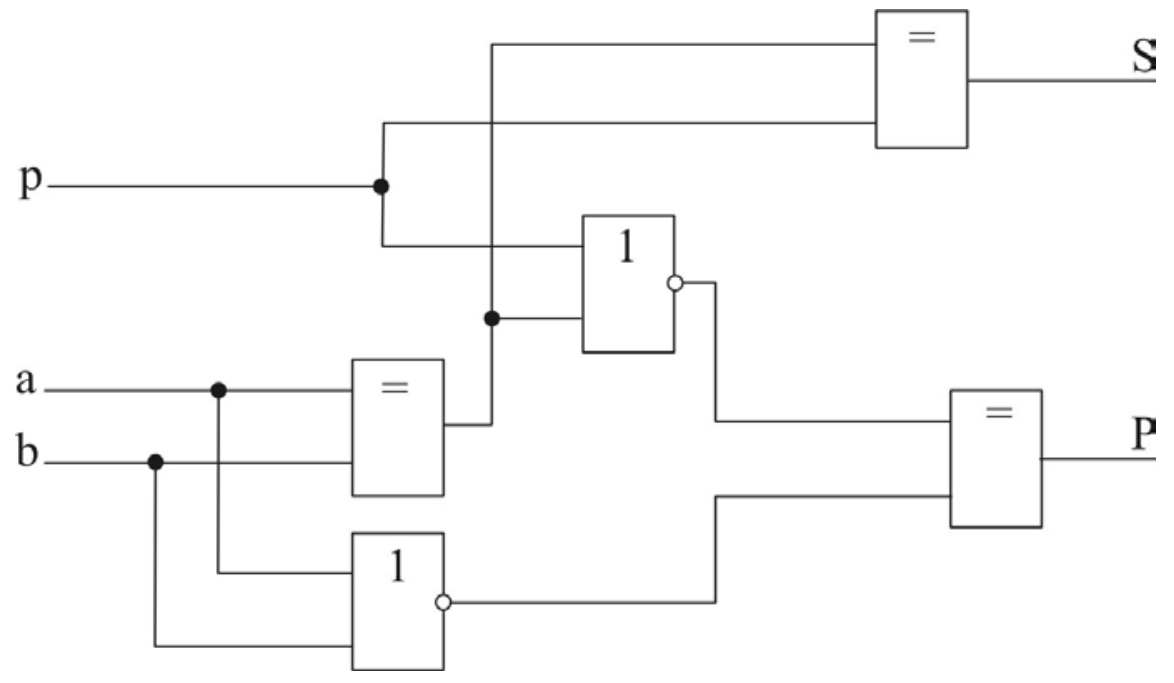

\begin{tabular}{l|c|c|c|c|c|}
\hline & $\mathrm{p}$ & $\mathrm{a}$ & $\mathrm{b}$ & $\mathrm{S}$ & $\mathrm{P}$ \\
\hline & 0 & 0 & 1 & 1 & 0 \\
\hline & 0 & 0 & 0 & 0 & 0 \\
\hline & 1 & 1 & 0 & 0 & 1 \\
\hline
\end{tabular}

Figure 1. Testable 1-bit adder circuit and its fault detection test

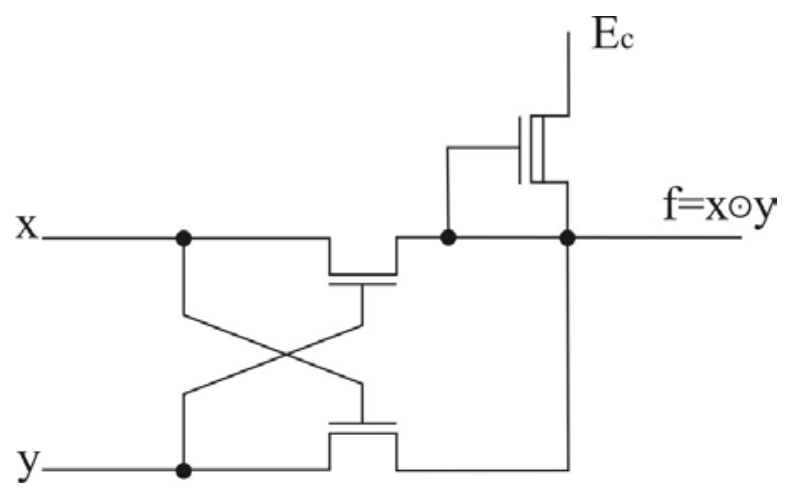

Figure 2. Schematic of EQUIVALENCE gate in MOS transistors

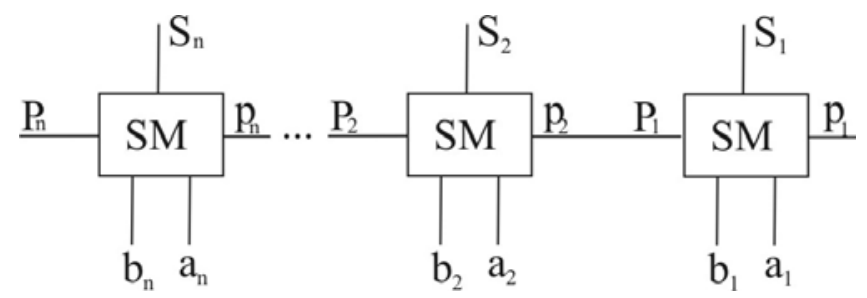

\begin{tabular}{|c|c|c|c|c|c|c|c|c|c|c|c|}
\hline$p_{1}$ & $b_{1}$ & $a_{1}$ & $b_{2}$ & $a_{2}$ & $b_{3}$ & $a_{3}$ & $\ldots$ & $S_{1}$ & $S_{2}$ & $S_{3}$ & $\ldots$ \\
\hline 0 & 1 & 0 & 1 & 0 & 1 & 0 & $\ldots$ & 1 & 1 & 1 & $\ldots$ \\
\hline 0 & 0 & 0 & 0 & 0 & 0 & 0 & $\ldots$ & 0 & 0 & 0 & $\ldots$ \\
\hline 1 & 0 & 1 & 0 & 1 & 0 & 1 & $\ldots$ & 0 & 0 & 0 & $\ldots$ \\
\hline
\end{tabular}

Figure 3. Testable $\mathrm{n}$-bit adder circuit and its fault detection test 


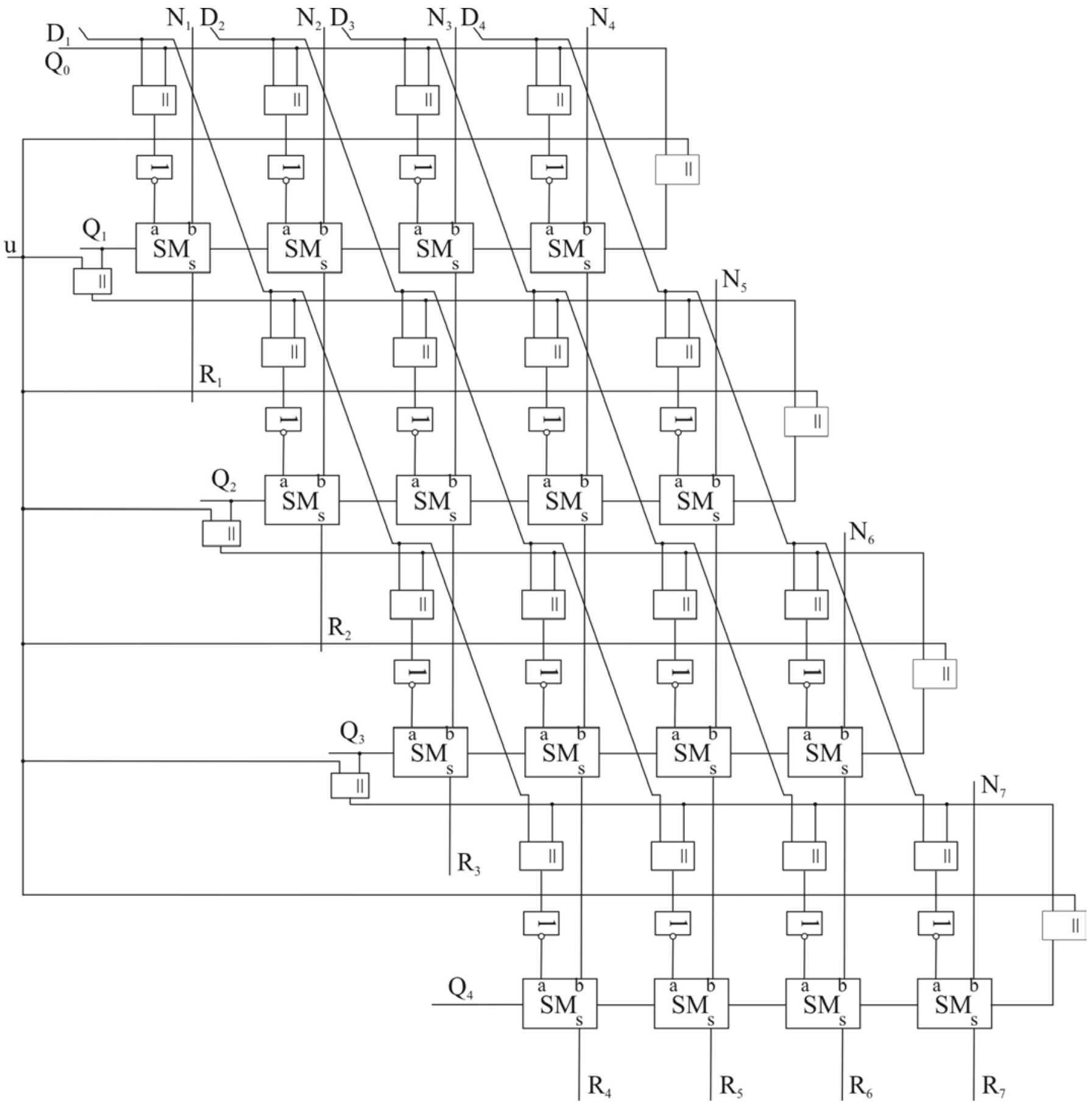

\begin{tabular}{|c|c|c|c|c|c|c|c|c|c|c|c|c|c|c|c|c|c|c|c|c|c|c|c|c|}
\hline & $\mathrm{u}$ & $\mathrm{Q}_{6}$ & $\mathrm{D}_{1}$ & $\mathrm{D}_{2}$ & $\mathrm{D}_{3}$ & $\mathrm{D}_{4}$ & $\mathrm{~N}_{1}$ & $\mathrm{~N}_{2}$ & $\mathrm{~N}_{3}$ & $\mathrm{~N}_{4}$ & $\mathrm{~N}_{5}$ & $\mathrm{~N}_{6}$ & $\mathrm{~N}_{7}$ & $\mathrm{Q}_{1}$ & $\mathrm{Q}_{2}$ & $\mathrm{Q}_{3}$ & $\mathrm{Q}_{4}$ & $\mathrm{R}_{1}$ & $\mathrm{R}_{2}$ & $\mathrm{R}_{3}$ & $\mathrm{R}_{4}$ & $\mathrm{R}_{5}$ & $\mathrm{R}_{6}$ & $\mathrm{R}_{7}$ \\
\hline & 0 & 1 & 1 & 1 & 1 & 1 & 1 & 1 & 1 & 1 & 1 & 1 & 1 & 0 & 0 & 0 & 0 & 1 & 1 & 1 & 1 & 1 & 1 & 1 \\
\hline & 1 & 0 & 0 & 0 & 0 & 0 & 0 & 0 & 0 & 0 & 0 & 0 & 0 & 0 & 0 & 0 & 0 & 0 & 0 & 0 & 0 & 0 & 0 & 0 \\
\hline 3 & 1 & 1 & 0 & 0 & 0 & 0 & 0 & 0 & 0 & 0 & 0 & 0 & 0 & 1 & 1 & 1 & 1 & 0 & 0 & 0 & 0 & 0 & 0 & 0 \\
\hline
\end{tabular}

Figure 4. Testable circuit of $4 \times 4$-bit nonrestoring array divider and its fault detection test

The testable functional-logical circuit of the $n=4$ is shown in (Fig. 4). In Fig. 4 nonrestoring $n \times n$ bit array divider for array with $\left(N_{1}, N_{2}, N_{3}, N_{4}, N_{5}, N_{6}, N_{7}\right)$ and $\left(D_{1}, D_{2}, D_{3}, D_{4}\right)$ 
represent the dividend and the divisor, respectively. $N_{1}$ and $D_{1}$ are the respective sign bits. $\left(Q_{1}, Q_{2}, Q_{3}, Q_{4}\right)$ are the quotient bits. $\left(R_{1}, R_{2}, R_{3}, R_{4}, R_{5}, R_{6}, R_{7}\right)$ are the remainder bits. The value of $Q_{i-1}$ determines whether subtraction or addition should be performed in the ith row. Since the initial subtraction must always be carried out, $Q_{0}=1$. The considered circuit contains seven additional two-input EQUIVALENCE gates and one additional input $u$. In this case, in the operating mode, the logical (1) signal is fed to the input $u$. The fault detection test for all single stuck-at faults of this circuit contains 3 vectors and is described in table shown in (Fig. 4).
The fault-detection test for the general case also contains 3 vectors and is constructed as follows:

- the sequence $1,0,0(j \in\{1,2, \ldots, n\})$ is fed to each $D_{j}$ input;

- the sequence $1,0,0(i \in\{1,2, \ldots, 2 n-1\})$ is fed to each $N_{i}$ input;

- the sequence $1,0,1$ is fed to $Q_{0}$ input, and the sequence $0,1,1$ is fed to $u$ input.

It is easily seen that in general the testable $n \times n$ bit nonrestoring array divider contains $2 \mathrm{n}-1$ additional two-input EQUIVALENCE gates. The testable circuit found in [1] requires significantly bigger amount of hardware resources for its implementation than the proposed testable circuit.

\section{References:}

1. Qiao Tong, Niraj K. Jha. Design of C-testable DCVS Binary Array Dividers // IEEE Journal of SolidState Circuits,- Vol. 26.- No. 2.- February 1991.- P. 134-141.

2. Яблонский С. В. Введение в дискретную математику.- М.: «Наука». Гм. реА. физ.-мат. мит. 1986.$384 \mathrm{c}$.

3. Мурога С. Системное проектирование сверхбольших интегральных схем. Кн. 1.- М.: Мир, 1985.$288 \mathrm{c}$. 